\title{
4D-STEM Imaging With the pnCCD (S)TEM-Camera
}

\author{
M. Simson ${ }^{1}$, H. Ryll², H. Banba ${ }^{3}$, R. Hartmann², M. Huth², S. Ihle², L. Jones ${ }^{4}$, Y. Kondo ${ }^{3}$, K. Müller ${ }^{5}$, \\ P.D. Nellist ${ }^{4}$, R. Sagawa ${ }^{3}$, J. Schmidt ${ }^{2}$, H. Soltau ${ }^{1}$, L. Strüder ${ }^{2}$ and H. Yang ${ }^{4}$ \\ 1. PNDetector GmbH, Sckellstraße 3, 81667 München, Germany \\ 2. PNSensor GmbH, Otto-Hahn-Ring 6, 81739 München, Germany \\ 3. JEOL Ltd.,3-1-2 Musashino Akishima Tokyo 196-8558, Japan \\ 4. Department of Materials, University of Oxford, 13 Parks Road, Oxford OX13PH, UK \\ 5. Universität Bremen, Otto-Hahn-Allee 1, 28359 Bremen, Germany
}

4D-STEM imaging describes a powerful imaging technique where a two-dimensional image is recorded for each probe position of a two-dimensional STEM image. For typical STEM images of 256x256 probe positions, a total of $65,5362 \mathrm{D}$ images needs to be recorded. This amount of data can be recorded with the pnCCD (S)TEM camera in a practical timeframe. This camera uses a direct detecting, radiation hard pnCCD with a minimum readout speed of 1000 full frames per second (fps) [1]. With the pnCCD (S)TEM camera a 4D data cube consisting of 256x256 probe positions with a 264x264 pixel detector image for each probe position can be recorded in less than $70 \mathrm{~s}$. Several measurements have been performed to prove the capability of the camera for 4D-STEM imaging, among them strain analysis, magnetic domain mapping and most recently electron ptychography.

Electron ptychography is a 4D-STEM technique that has been described theoretically already in 1993 [2] but so far was limited experimentally by the low readout speed of existing cameras. In this technique, the intensity distribution in the bright field disk is recorded in 2D for each STEM probe position (Figure 1a). In an electron wave-optical approach the phase and amplitude information is extracted from the recorded intensity images. The reconstructed phase image shows enhanced image contrast compared to the conventional annular dark field image (Figure 2). Measurements with the pnCCD (S)TEM camera were carried out using a JEOL ARM200-CF in order to investigate different samples with the ptychographic phase reconstruction technique. Simulations show that the phase reconstruction technique does not require the full camera resolution of $264 \times 264$ pixels [3]. The pnCCD camera can be used in windowing and binning modes to further increase the measurement speed. Different combinations were tested at up to $4000 \mathrm{fps}$ (4-fold binning in one direction, i.e. 66x264 pixels, see Figure 1c). A 512x512 STEM image can then be recorded in just over a minute.

Other 4D-STEM applications like strain analysis or magnetic domain mapping use the cameras's ability to precisely determine the exact position of the bright field disk on the detector. When traversing the sample the electron beam can be deflected due to different effects in the sample causing a movement of the BF disk's position. The advantage of using a 2D detector over conventional quadrant DPC detectors is the possibility to separate movements of the disk from intensity fluctuations inside it. Strain in the sample causes such movements and can be measured by 4D-STEM [4]. Imaging of magnetic fields inside the sample is possible using a Lorentz-like setup of the STEM, a 2D detector and analysis of the movements of the BF disk. Measurements demonstrating the technique with a nickel sample were done.

Also dark field measurements profit from using 4D-STEM. By adapting the camera length, the 2D diffraction pattern can be recorded. In an offline analysis different STEM images can be reconstructed 
by using different parts of the detector images corresponding to different parts of the scattered beam.

In conclusion we have shown that with the pnCCD camera new techniques in STEM are promoted and various fields of application benefit from recording the two-dimensional detector image. With its direct detection, high readout speed and radiation hardness the pnCCD (S)TEM camera makes recording of large 4D data cubes in short times possible and enables new science.

[1] H. Ryll et al., Microscopy and Microanalysis 19 (2013), p.1160-1161.

[2] J.M. Rodenburg, B.C. McCallum, P.D. Nellist, Ultramicroscopy. 48 (1993) 304-314.

[3] H. Yang et al., Ultramicroscopy. doi:10.1016/j.ultramic.2014.10.013

[4] K. Müller et al., Appl. Phys. Lett. 101 (2012), p. 2121101-2121104.
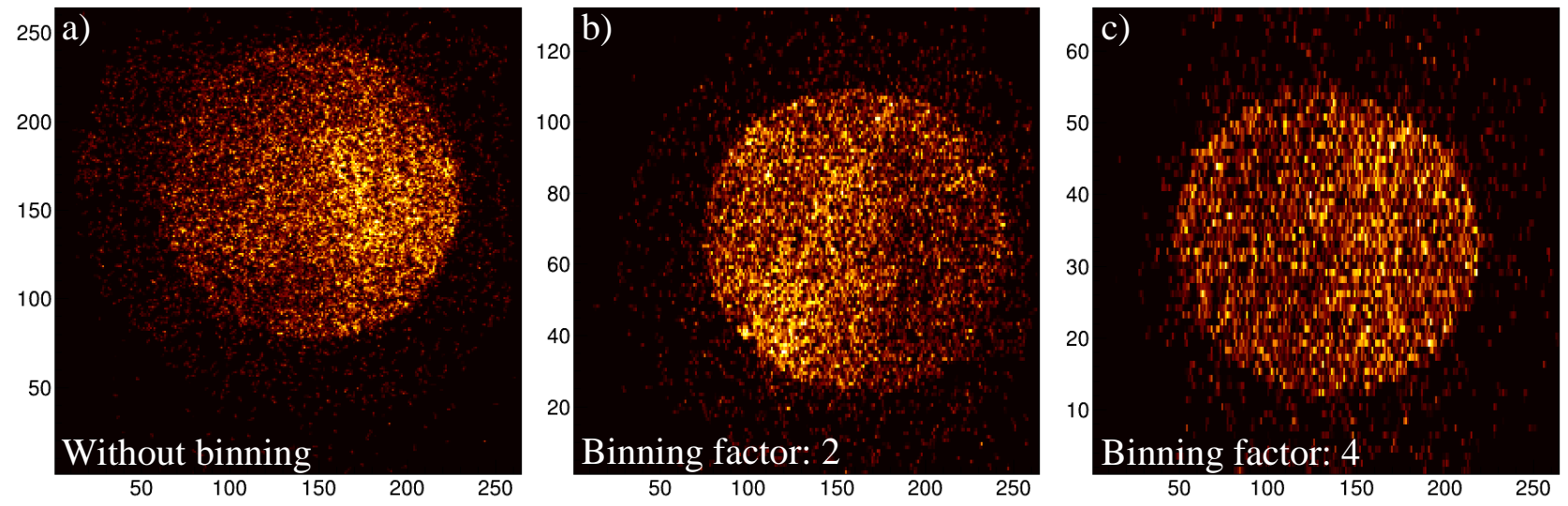

Figure 1. Examples of recorded single raw images of the bright field disk for individual probe positions in STEM. Images were recorded without binning (a), 2-fold binning (b) and 4-fold binning (c). Pixel dwell times were $1000 \mu \mathrm{s}, 500 \mu \mathrm{s}$ and $250 \mu \mathrm{s}$, respectively. The intensity distributions in these images are analyzed to extract the phase information.
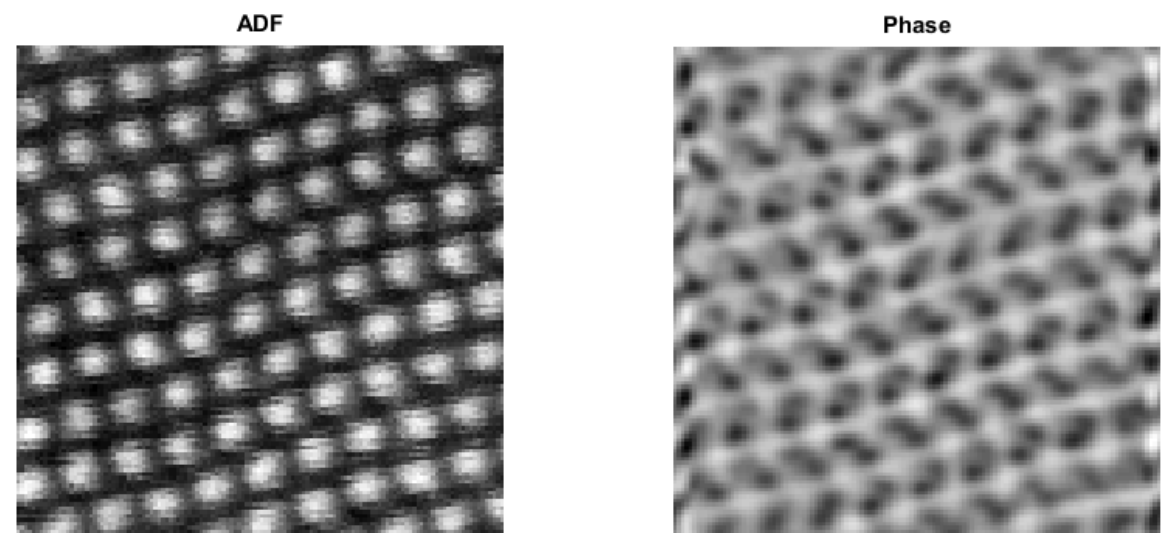

Figure 2. Comparison of the conventional annular dark field (ADF) image and the reconstructed phase of a GaN sample using a ptychography method. Pairs of Ga-N columns show in the reconstructed phase, but the $\mathrm{N}$ columns are nearly invisible in the simultaneously recorded ADF image. Images contain $128 \times 128$ probe positions recorded at a dwell time of $250 \mu \mathrm{s}$. 